\title{
Clinical Characteristics, Treatment Effectiveness, and Predictors of Response to Pharmacotherapeutic Interventions Among Patients with Herpetic-Related Neuralgia: A Retrospective Analysis
}

\author{
Jiehua Zhou • Wuping Sun · Yingzhi Liu · Shaomin Yang · Songbin Wu · Sashuang Wang • \\ Donglin Xiong · Idy Hiuting Ho • Lin Zhang • Matthew Tak Vai Chan • William Ka Kei Wu • \\ Xiaodong Liu $\cdot$ Lizu Xiao
}

Received: June 14, 2021 / Accepted: August 5, 2021 / Published online: September 12, 2021

(C) The Author(s) 2021

\section{ABSTRACT}

Background: The treatment for herpetic-related neuralgia focuses on symptom control by use of antiviral drugs, anticonvulsants, and tricyclic antidepressants. We aimed to explore the clinical characteristics associated with medication responsiveness, and to build a classifier for

Jiehua Zhou and Wuping Sun contributed equally to this work.

Supplementary Information The online version contains supplementary material available at https:// doi.org/10.1007/s40122-021-00303-7.

J. Zhou $\cdot$ W. Sun $\cdot$ S. Yang $\cdot$ S. Wu $\cdot$ S. Wang

D. Xiong · L. Xiao $(\bowtie)$

Shenzhen Municipal Key Laboratory for Pain

Medicine, Department of Pain Medicine, Shenzhen

Nanshan People's Hospital, The 6th Affiliated

Hospital of Shenzhen University Health Science

Center, National Key Clinical Pain Medicine of

China, Shenzhen 518060, China

e-mail: nsyyjoe@live.cn

Y. Liu · I. H. Ho · L. Zhang · M. T. V. Chan .

W. K. K. Wu X. Liu ( $₫)$

Department of Anaesthesia and Intensive Care, The Chinese University of Hong Kong, Sha Tin, Hong

Kong SAR, China

e-mail:xdliu@cuhk.edu.hk

Y. Liu · I. H. Ho · L. Zhang · M. T. V. Chan .

W. K. K. Wu . X. Liu

Peter Hung Pain Research Institute, The Chinese

University of Hong Kong, Sha Tin, Hong Kong SAR,

China identification of patients who have risk of inadequate pain management.

Methods: We recruited herpetic-related neuralgia patients during a 3-year period. Patients were stratified into a medication-resistant pain (MRP) group when the pain decrease in the visual analogue scale (VAS) is $<3$ points, and otherwise a medication-sensitive pain (MSP) group. Multivariate logistic regression was performed to determine the factors associated with MRP. We fitted four machine learning (ML) models, namely logistic regression, random forest, supporting vector machines (SVM), and naïve Bayes with clinical characteristics gathered at admission to identify patients with MRP. Results: A total of 213 patients were recruited, and 132 (61.97\%) patients were diagnosed with MRP. Subacute herpes zoster (HZ) (vs. acute, OR 8.95, 95\% CI 3.15-29.48, $p=0.0001$ ), severe lesion (vs. mild lesion, OR 3.84, 95\% CI 1.44-10.81, $p=0.0084$ ), depressed mood (unit increase OR 1.10, 95\% CI 1.00-1.20, $p=0.0447$ ), and hypertension (hypertension, vs. no hypertension, OR $0.36,95 \%$ CI $0.14-0.87, p=0.0266$ ) were significantly associated with MRP. Among four ML models, SVM had the highest accuracy (0.917) and receiver operating characteristic-area under the curve (0.918) to discriminate MRP from MSP. Phase of disease is the most important feature when fitting ML models.

Conclusions: Clinical characteristics collected before treatment could be adopted to identify 
patients with MRP.Keywords: Herpes zoster; Herpetic-related neuralgia; Neuropathic pain; Machine learning

\section{Key Summary Points}

In the present study, we identified the phase of disease, the degree of lesion, hypertension, and depression-associated mood as potential predictors for treatment responsiveness in herpetic-related neuralgia patients. Subacute HZ patients with server skin lesions and depressed mood had higher risks for treatment resistance. By contrast, patients with hypertension were associated with better drug responsiveness than those who were normotensive. Furthermore, we fitted logistic regression, RF, SVM, and NB machine learning models to identify MRP by inputting basic clinical characteristics. We found that the SVM model had the highest accuracy to discriminate MRP from MSP, with a prediction accuracy comparable to previous imaging or EEG-based approaches.

\section{INTRODUCTION}

Herpes zoster (HZ) is caused by the reactivation of varicella-zoster virus (VZV) among $\mathrm{T}$ lymphocytes in the dorsal root ganglions [1]. The initial symptoms of $\mathrm{HZ}$ include headache, fever, and altered skin sensation. This is followed by the development of painful maculopapular rashes and blisters along the dermatomal distribution for weeks to months [2-4]. It was estimated that the incidence of $\mathrm{HZ}$ is around $7.7 \%$ in China, with a lifetime risk of $30 \%$ in individual [5]. Previous studies have identified risk factors such as malignant diseases, immunodeficiencies, autoimmune diseases, mental disorders, and use of immunosuppressive therapies as being associated with VZV reactivation [6]. In this respect, a decrease in cell-mediated immunity increases the risk of VZV reactivation 100 -fold. Up to $30 \%$ patients with HZ developed postherpetic neuralgia (PHN), producing moderate-to-severe pain for years and seriously affecting the quality of life for the patients and their families.

The treatment regimens of herpetic-related neuralgia involve the use of topical lidocaine/capsaicin, gabapentin, pregabalin, and tricyclic antidepressants according to the guideline of PHN treatment [7-9]. In addition, patients often receive antiviral medications to improve immunity $[10,11]$. The efficacies for these regimens are, however, limited. Therefore, patients choose minimally invasive treatment rather than continuing to take much more medications after several days' medication treatment. However, the factors that affect patient responsiveness to pharmacological treatment are still unclear.

Previous study has proposed that combined functional magnetic resonance imaging (fMRI) and multivoxel pattern analysis (MVPA) analysis might be adopted to predict treatment outcome [12]. In addition, by analyzing EEG data, features of beta-band power spectral entropy in the central-parietal region could be selected to build a classifier separating HZ patients with different drug responsiveness [13]. While these medical tests might assist clinical decisions with considerable prediction accuracy $(80 \% \pm 11.7 \%)$, specificity $(77.7 \% \pm 27.3 \%)$, and sensitivity $(82.5 \% \pm 14.7 \%)$, they are not readily accessible to all the patients due to the cost and clinical resources concerns. The present study aimed to explore the clinical characteristics associated with treatment success using a machine learning method. In this study, we found that the phase of diseases, degree of skin lesion, and depression are independent risk factors, whereas hypertension is a potential protective factor for developing analgesic resistance among patients with herpetic-related neuralgia. Besides, an SVM model adopting basic clinical features at admission could predict the outcome of analgesic responsiveness. 


\section{METHODS}

\section{Patient recruitment and clinical information collection}

This was a retrospective study. The present study was conducted in accordance with the Declaration of Helsinki and approved by the ethics committee of Shenzhen Nanshan People's Hospital and the 6th Affiliated Hospital of Shenzhen University Health Science Center (No. 2016041201). The subjects were HZ patients who met the inclusion and exclusion criteria for this study. The inclusion criteria included HZ-related persistent pain Visual Analog Scale (VAS) $\geq 5$ scores, aged $50-80$ years old, and accepted treatment in Shenzhen Nanshan People's Hospital and the 6th Affiliated Hospital of Shenzhen University Health Science Center from January 2017 to January 2020. The exclusion criteria included $\mathrm{HZ}$ patients with mental illness, serious cardiocerebrovascular or hepatorenal insufficiency, disease related to the hypothalamic-pituitary-adrenal cortical axis (HPA axis), other infectious diseases (such as tuberculosis and AIDS), hormone therapy within 2 months before admission (such as dexamethasone, hydrocortisone, and methylprednisolone).

We collected baseline patient information at baseline, including sex, age, phase of disease (acute HZ within 1 month of the onset, subacute $\mathrm{HZ}$ within 3 months of the onset, or postherpetic neuralgia with more than 3 months of the onset), pain intensity, extent of skin lesions (mild skin lesions with less than $1 / 3$ related dermatome area, moderate skin lesions with more than $1 / 3$ and less than $2 / 3$ related dermatome area, or severe skin lesions with more than 2/3 related dermatome area), complications, family history of disease, and affected regions (cervical, thoracic, lumbar, or sacral segments). Patients also completed a series of self-reporting measures of pain, depression, anxiety, and sleep quality using VAS score, simple McGill pain questionnaire (MPQ), patient health questionnaire 9 (PHQ9), StateTrait Anxiety Inventory (STAI), and Pittsburgh Sleep Quality Index (PSQI).

\section{Pain management and patient subgrouping}

All the medications prescribed to the patients were according to the guidelines of PHN treatment [9]: gabapentin therapy with initial dose 100-300 mg three times daily; increase dose by 100-300 mg three times every 1-7 days as tolerated up to a maximum of $3600 \mathrm{mg} /$ day unless the patient has a serious side effect; pregabalin therapy with initial dose 75-150 mg/day, maximum $600 \mathrm{mg} /$ day, 1-3 times/day; and amitriptyline treatment with initial dose $10 \mathrm{mg} /$ day, maximum $50 \mathrm{mg} /$ day, once daily before bedtime. Two weeks after treatment, patients reported pain scores using the VAS. We classified patients as treatment-sensitive if there were three points or more in reduction in VAS score after pharmacological therapy. Those with $<3$ points reduction in VAS score were considered as treatment-resistant, as previously reported [14].

\section{Bivariate and multivariate logistic regression}

The association between treatment responsiveness and baseline (demographic and clinical) characteristics was determined by binary logistic regression [15]. For continuous variables, normality and homogeneity of variance were tested by Shapiro test

and Levene's test, respectively. The differences between groups were then compared using Mann-Whitney test. Variables with $p$ values $<0.1$ in the univariate analysis were included in the multivariate logistic regression analysis. A $p$ value $<0.05$ was considered statistically significant.

\section{Machine learning classifier}

We fitted logistic regression, random forests (RF), support vector machines (SVM), and naïve Bayes (NB) models to identify patients who were treatment responsive or not $[16,17]$. The dataset (213 patients $\times 16$ features) was randomly split into derivation set and validation set in a ratio of 75:25. The derivation set was used for 
modeling, and the validation set was used to validate the final model's performance. For logistic regression, the glm and step functions in $\mathrm{R}$ were adopted for model selection by Akaike Information Criterion (AIC) in a stepwise algorithm. The maximum number of steps were set to 1000 . To build an RF classifier, the Boruta package in $\mathrm{R}$ was firstly used to select relevant clinical variables, followed by RF modeling with the randomForest package. For SVM modeling, e1071 and Tidymodels package was used. We trained the model with linear and non-linear kernel (radial basis function and polynomial). The final SVM model was chosen based on the accuracy using validation set. We also fitted the NB model with $e 1071$ package using the default settings. To evaluate the performance of four classifiers, the receiver operating characteristics (ROC) curves were plotted with the $p R O C$ package and the areas under the curve (AUC) were calculated.

\section{RESULTS}

\section{Bivariate and multivariate logistic regression}

A total of $213 \mathrm{HZ}$ patients recruited during the period from January 2017 to January 2020 were included in the current study. Patient baseline characteristics are summarized in Table 1. Hypertension, diabetes mellitus, prior cancer (cancer), and coronary heart disease, which accounted for over $5 \%$ the participants in the current study, were extracted and analyzed independently. We prescribed all the patients pregabalin/gabapentin, and amitriptyline. Only 81 patients $(38.03 \%)$ had experienced satisfactory pain relief (reduction in VAS $>3$ ), while 132 patients $(61.97 \%)$ needed adjusted therapeutic strategies (for instance, nerve block, pulsed radiofrequency, and spinal cord stimulation). According to the outcome of treatment, 81 patients were classified as treatment-sensitive, and 132 patients were treatment-resistant. In the derivation set, older patients had a higher risk for treatment resistance (age 60-69 years vs. 50-59 years-odds ratio (OR) 2.29, 95\% confidence interval (CI) 1.13-4.70, $p=0.021 ; \geq 70$ years vs. $50-59$ years, OR 3.11 , 95\% CI 1.47-6.74, $p=0.0029$ ). In addition, male patients (OR 1.78, 95\% CI 1.01-3.14, $p=0.045)$, patients with higher extent of lesion (severe vs. mild, OR 3.62, 95\% CI 1.86-7.26, $p<0.001$ ) or later phase of disease (SHZ vs. AHZ, OR 6.05, 95\% CI 2.87-13.85, $p<0.001$; PHN vs. AHZ, OR 42.74, 95\% CI 8.84-1026.39, $p<0.0001)$ were prone to drug resistance (Table 1). Skin lesions that occurred in cervical segment (OR $0.32, \quad 95 \%$ CI $0.10-0.93$, $p=0.036$ ), cervical-thoracic segment (OR 0.23 , 95\% CI 0.06-0.85, $p=0.028$ ), and lower back (OR 0.10, 95\% CI 0.00-1.01, $p=0.0478$ ) were less likely than in face (innervated by the trigeminal nerve) to be resistant to medications. The differences in scores for pain (VAS, McGill), mood (PHQ9, and anxiety), and sleep quality (PSQI) were also compared with Mann-Whitney tests. Except for the relationship between PSQI and anxiety, all of the variables were mutually correlated, with rho ranging from 0.17 to 0.52 (Supplementary Fig. 1). As demonstrated in Fig. 1, patients with treatment resistance had higher McGill, PHQ9, and PSQI scores than those who responded to treatment. Patients with increased pain experience on admission (OR 1.05, 95\% CI 1.01-1.10, $p=0.033$ ), sleep problems (OR 1.07, 95\% CI 1.00-1.14, $p=0.037$ ), and depressed mood (OR 1.09, 95\% CI 1.03-1.16, $p=0.002$ ) had a higher risk of treatment resistance (Fig. 1A-E). Multivariate logistic regression analysis was then applied with variables with $p$ values $<0.05$. Severe lesion (vs. mild lesion, adjusted OR 3.84, 95\% CI 1.44-10.81, $p=0.008$ ), hypertension (vs. normotension, adjusted OR 0.36, 95\% CI $0.14-0.87, p=0.027$ ), subacute $\mathrm{HZ}$ (vs. acute HZ, adjusted OR 8.95, 95\% CI 3.15-29.48, $p<0.001)$ and depressed mood (adjusted OR $1.10,95 \%$ CI $1.00-1.20, p=0.045)$ were still significantly associated with higher odds of MRP (Table 1), implying that these characteristics might be determinants of patients' medication responsiveness. 
Table 1 Bivariate and multivariate analysis of clinical characteristics

\begin{tabular}{|c|c|c|c|c|c|c|}
\hline \multirow[t]{3}{*}{ Characteristic } & \multicolumn{4}{|c|}{ Univariate analysis } & \multicolumn{2}{|c|}{ Multivariate analysis } \\
\hline & \multicolumn{2}{|c|}{ Patients, $n(\%)$} & \multirow[t]{2}{*}{ OR $(95 \% \mathrm{CI})$} & \multirow[t]{2}{*}{$p$ value } & \multirow[t]{2}{*}{$\overline{\text { OR }(95 \% \mathrm{CI})}$} & \multirow[t]{2}{*}{$p$ value } \\
\hline & MSP & MRP & & & & \\
\hline \multicolumn{7}{|l|}{ Age } \\
\hline $50-59$ & $28(56.00)$ & $22(44.00)$ & - & - & - & \\
\hline $60-69$ & $32(35.56)$ & $58(64.44)$ & $2.29(1.13-4.70)$ & 0.0212 & $1.75(0.68-4.61)$ & 0.2470 \\
\hline$\geq 70$ & $21(28.77)$ & $52(71.23)$ & $3.11(1.47-6.74)$ & 0.0029 & $0.69(0.21-2.13)$ & 0.5246 \\
\hline \multicolumn{7}{|l|}{ Gender } \\
\hline Female & $49(44.55)$ & $61(55.45)$ & - & - & - & \\
\hline Male & $32(31.07)$ & $71(68.93)$ & $1.78(1.01-3.14)$ & 0.0445 & $1.56(0.66-3.74)$ & 0.3102 \\
\hline \multicolumn{7}{|l|}{ Lesion } \\
\hline Mild & $42(52.50)$ & $38(47.50)$ & - & - & - & - \\
\hline Moderate & $20(39.22)$ & $31(60.78)$ & $1.70(0.84-3.53)$ & 0.1434 & $1.33(0.45-3.88)$ & 0.6012 \\
\hline Severe & $19(23.17)$ & $63(76.83)$ & $3.62(1.86-7.26)$ & 0.0001 & $3.84(1.44-10.81)$ & 0.0084 \\
\hline \multicolumn{7}{|c|}{ High blood pressure } \\
\hline No & $52(34.43)$ & $99(65.56)$ & - & - & & - \\
\hline Yes & $29(46.77)$ & $33(53.23)$ & $0.60(0.33-1.10)$ & 0.0972 & $0.36(0.14-0.87)$ & 0.0266 \\
\hline \multicolumn{7}{|l|}{ Diabetes } \\
\hline No & $73(39.67)$ & $111(60.33)$ & - & - & & \\
\hline Yes & $8(27.59)$ & $21(72.41)$ & $1.70(0.74-4.33)$ & 0.2195 & & \\
\hline \multicolumn{7}{|l|}{ CHD } \\
\hline No & $73(37.82)$ & $120(62.18)$ & - & - & & \\
\hline Yes & $8(39.60)$ & $12(60.40)$ & $0.91(0.35-2.45)$ & 0.8423 & & \\
\hline \multicolumn{7}{|l|}{ Cancers } \\
\hline No & $78(38.24)$ & $126(61.76)$ & $0.83(0.16-3.34)$ & 1.0000 & & \\
\hline Yes & $3(33.33)$ & $6(66.67 \times)$ & & & & \\
\hline \multicolumn{7}{|c|}{ Prior antiviral drugs } \\
\hline No & $41(36.61)$ & $71(63.39)$ & - & - & & \\
\hline Yes & $40(40.00)$ & $61(60.00)$ & $0.88(0.50-1.54)$ & 0.8812 & & \\
\hline \multicolumn{7}{|l|}{ Family history } \\
\hline Yes & $72(37.11)$ & $122(62.89)$ & - & - & & \\
\hline No & $9(47.37)$ & $10(52.63)$ & $0.66(0.25-1.75)$ & 0.3918 & & \\
\hline \multicolumn{7}{|l|}{ Location } \\
\hline V1-3 & $6(20.00)$ & $24(80.00)$ & - & - & - & \\
\hline
\end{tabular}


Table 1 continued

\begin{tabular}{|c|c|c|c|c|c|c|}
\hline \multirow[t]{3}{*}{ Characteristic } & \multicolumn{4}{|c|}{ Univariate analysis } & \multicolumn{2}{|l|}{ Multivariate analysis } \\
\hline & \multicolumn{2}{|c|}{ Patients, $n$ (\%) } & \multirow[t]{2}{*}{ OR $(95 \% \mathrm{CI})$} & \multirow[t]{2}{*}{$p$ value } & \multirow[t]{2}{*}{$\overline{\mathrm{OR}}(95 \% \mathrm{CI})$} & \multirow[t]{2}{*}{$p$ value } \\
\hline & $\overline{\text { MSP }}$ & MRP & & & & \\
\hline $\mathrm{C} 1-8$ & $17(44.74)$ & $21(5.26)$ & $0.32(0.10-0.93)$ & 0.0360 & $0.28(0.06-1.12)$ & 0.0783 \\
\hline $\mathrm{T} 1-12$ & $42(37.17)$ & $71(62.83)$ & $0.43(0.15-1.09)$ & 0.0775 & $0.53(0.15-1.74)$ & 0.3083 \\
\hline $\mathrm{C} 1-\mathrm{T} 12$ & $9(52.94)$ & $8(47.06)$ & $0.23(0.06-0.85)$ & 0.0276 & $0.43(0.07-2.34)$ & 0.3346 \\
\hline L1-5 & $3(75.00)$ & $1(25.00)$ & $0.10(0.00-1.01)$ & 0.0478 & $0.42(0.02-6.22)$ & 0.5359 \\
\hline S1-5 & $1(33.33)$ & $2(66.67)$ & $0.49(0.03-17.11)$ & 0.5235 & $0.46(0.01-25.21)$ & 0.7015 \\
\hline $\mathrm{L} 1-\mathrm{S} 5$ & $3(37.50)$ & $5(62.50)$ & $0.42(0.07-2.71)$ & 0.3631 & $0.60(0.04-10.03)$ & 0.7104 \\
\hline \multicolumn{7}{|l|}{ Phase } \\
\hline $\mathrm{AHZ}$ & $70(57.85)$ & $51(42.15)$ & - & - & & \\
\hline SHZ & $10(18.18)$ & $45(81.82)$ & $6.05(2.87-13.85)$ & $<0.0001$ & $8.95(3.15-29.48)$ & 0.0001 \\
\hline PHN & $1(2.70)$ & $36(97.30)$ & $42.74(8.84-1026.39)$ & $<0.0001$ & $2.37 \mathrm{e}^{8}\left(6.57 \mathrm{e}^{-9}-1.45 \mathrm{e}^{160}\right)$ & 0.9845 \\
\hline VAS & - & - & $0.95(0.78-1.16)$ & 0.5990 & & \\
\hline McGill & - & - & $1.05(1.01-1.10)$ & 0.0328 & & \\
\hline PSQI & - & - & $1.07(1.00-1.14)$ & 0.0369 & & \\
\hline PHQ9 & - & - & $1.09(1.03-1.16)$ & 0.0023 & $1.10(1.00-1.20)$ & 0.0447 \\
\hline Anxiety & - & - & $1.01(0.99-1.03)$ & 0.2130 & & \\
\hline
\end{tabular}

\section{Machine learning identification of MRP}

We have fitted four machine learning models to identify MRP. In the logistic regression model, a backward stepwise algorithm was applied to determine the features that might significantly affect the performance of classifier. The model containing four variables, namely lesion, hypertension, diabetes mellitus, and stage of disease, had the lowest AIC. This result implied that these four clinical characteristics together might be used to predict treatment resistance. By using a validation set, we found that the prediction accuracy was 0.833 . To fit the RF model, the Boruta package was firstly applied to select features. After performing 99 interactions, four features, namely age, anxiety, lesion extent, and phase of disease were considered important, and two features, namely hypertension and McGill scores, were regarded as tentative attributes (Supplementary Fig. 2). The other features, including prior antiviral treatment, family history, and "have diabetes", were confirmed unimportant (Supplementary Fig. 2). We then selected four important and two tentative features to fit the RF model, which discriminated treatment success with treatment resistance with an accuracy of 0.750 . For the NB model, the e1071 package was applied with all the variables. The model has an accuracy of 0.771 using the test cohort. To build a SVM classifier, we have trained the derivation set with linear and radial basis function kernels. For parameter tuning, cross-validation was performed using the grid search method. The mean prediction accuracies of linear, radial basis function non-linear, and polynomial non-linear SVM were $0.878,0.714$, and 0.917 , respectively. Therefore, polynomial kernel was chosen to fit 

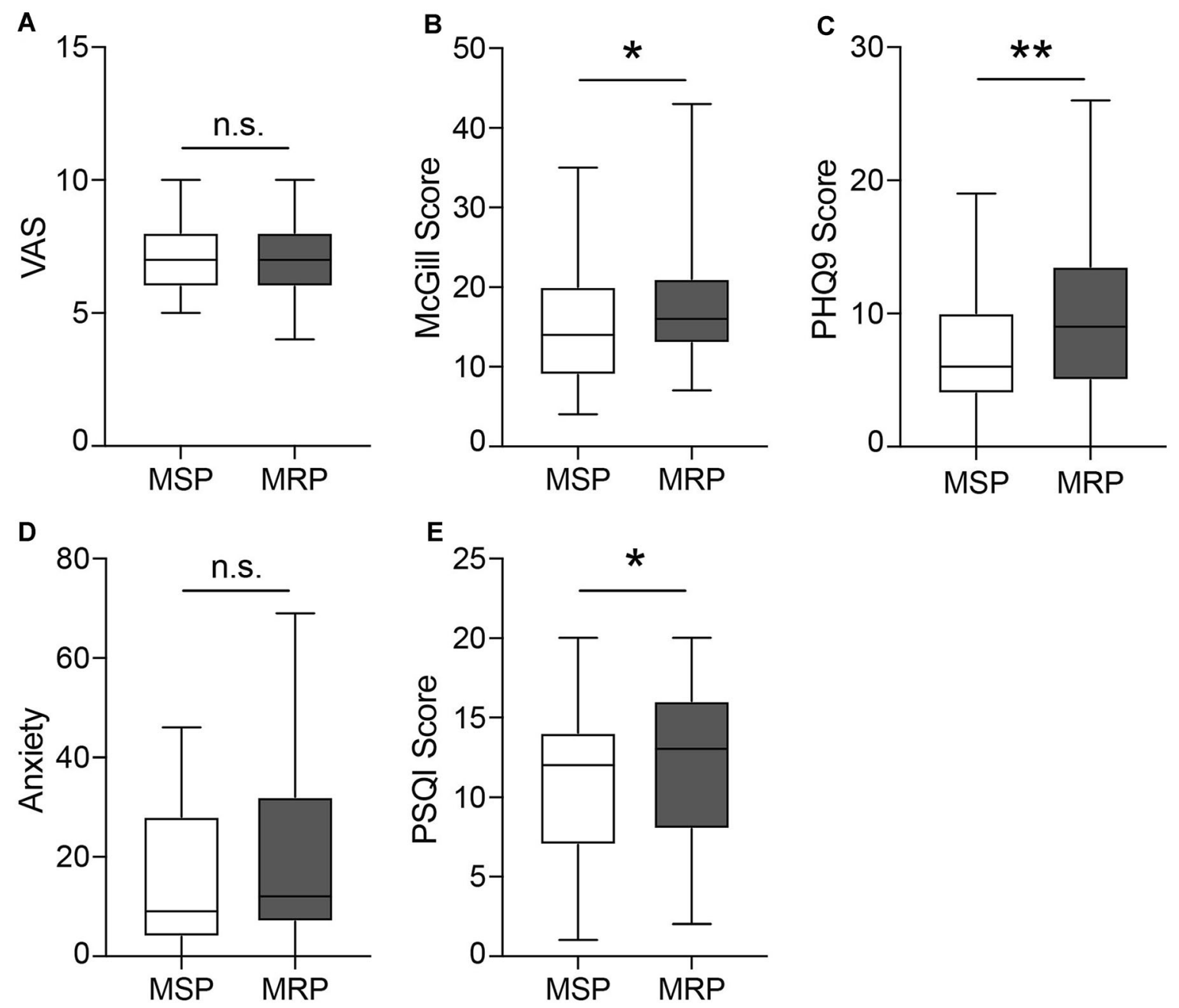

Fig. 1 Questionnaire items of herpetic-related neuralgia patients between MSP and MRP groups. The differences in scores for VAS (A), McGill (B), depression (PHQ9, C), anxiety (D), and sleep quality (PSQI, E) between MSP and

the SVM model. Finally, we plotted the ROCAUC of all four models (Fig. 2A). In terms of AUC, we ranked SVM (AUC: 0.918, 95\% CI 0.819-1.018, $p<0.001)$ the best model, following with RF (AUC: 0.826, 95\% CI $0.707-0.946, \quad p<0.001)>$ logistic regression (GLM) (AUC: 0.818, 95\% CI 0.686-0.950, $p<0.001)>\mathrm{NB} \quad$ (AUC: $0.797, \quad 95 \% \quad \mathrm{CI}$ $0.670-0.923, p<0.001)$. The variables importance plot (VIP) analysis of SVM suggested that phase of disease, diabetes, degree of lesion, location, depressed mood, family history, and hypertension were the top critical features in
MRP groups before treatment. Mean \pm SEM, ${ }^{*} p<0.01$, and ${ }^{* *} p<0.01$. n.s. no significant difference. Mann-Whitney tests. $M S P$ medical sensitive patients, MRP medical resistance patients

the diagnosis of MRP (Fig. 2B). Therefore, by simply using the clinical characteristics collected at admission, we may build a machine learning model to identify patients with MRP. This model has a prediction accuracy (0.917), which is superior to previous imaging or EEGbased models.

\section{DISCUSSION}

In this study, we identified the phase of disease, the degree of lesion, hypertension, and 

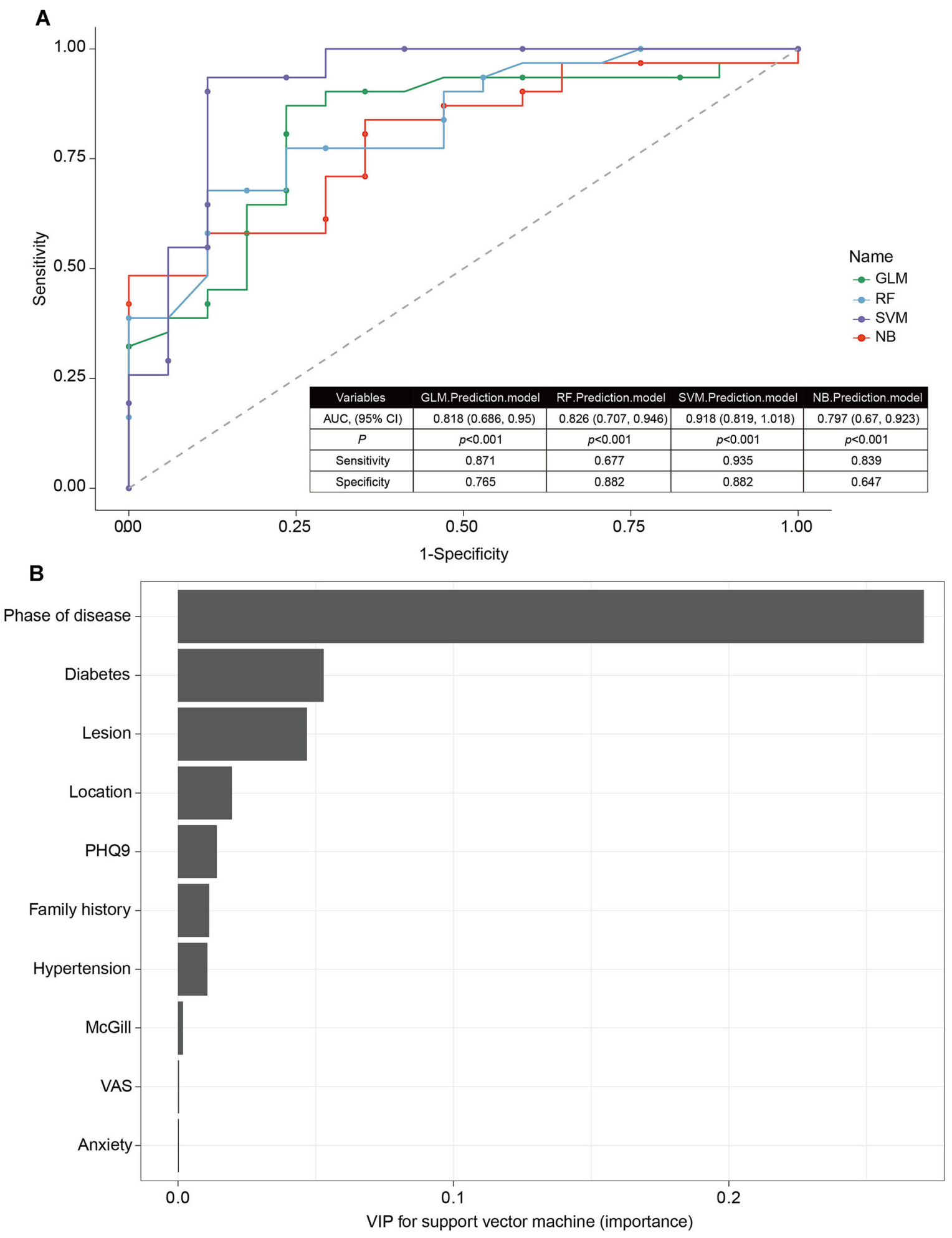

Fig. 2 Machine learning identification of MRP. ROCAUC of four models, including GLM prediction model, prediction model (A). The variables importance plot (VIP) RF prediction model, SVM prediction model, and NB analysis of SVM prediction model (B) 
depression-associated mood as potential predictors for treatment responsiveness in herpeticrelated neuralgia patients. Subacute HZ patients with server skin lesions and depressed mood had higher risks for treatment resistance. By contrast, patients with hypertension were associated with better drug responsiveness than those who were normotensive. Furthermore, we fitted logistic regression, RF, SVM, and NB machine learning models to identify MRP by inputting basic clinical characteristics. We found that the SVM model had the highest accuracy to discriminate MRP from MSP, with a prediction accuracy comparable to previous imaging or EEG-based approaches [13, 18].

Clinical characteristics of patients with herpetic-related neuralgia are associated with the effectiveness of pain medications. The multivariate analysis revealed four factors significantly associated with the outcome of analgesics. Among these characteristics, the phase of diseases, i.e., acute, subacute, or chronic (postherpetic neuralgia) and degree of skin lesion appeared to impact the outcome of medications most dramatically. For instance, more than half of the patients with acute $\mathrm{HZ}$ (57.85\%) benefitted from oral pain analgesics, whereas $81.82 \%$ patients with subacute $\mathrm{HZ}$ were still suffering from pain at the similar levels as prior treatment. This result implied that patients who immediately seek medical care at the sign of rashes might have better outcome of analgesics treatment. For PHN patients, the odds of probability to have poor drug responsiveness were as high as 42.74 (95\% CI 8.84-1026.39, $p<0.0001$ ) when compared with acute HZ patients. However, there was no significant association between PHN and poor responsiveness in the multivariate analysis, indicating that PHN was not an independent risk factor of MRP. Indeed, only $2.70 \%$ of patients with PHN benefit from an anticonvulsant in this cohort study. The response rate is inconsistent with previous reviews that gabapentin [19] and pregabalin [20] may reduce pain by a third in about 50\% patients with PHN. This observation reinforced the idea that PHN interacted with other characteristics to affect the responsiveness of analgesics. Various chronic pain conditions were associated with high incidence of comorbidities, including depression, anxiety, and sleep disorders [21]. A meta-analysis among 237,952 people demonstrated that compared with no depression, people with depressive episodes were associated with 2.11 times higher likelihood of having severe pain [22]. On the other hand, up to $60 \%$ patients with chronic pain had depressive episodes, and more than $30 \%$ of these patients had severe depression [23]. The current analysis suggested that these pain comorbidities might also affect medication responsiveness. Negative mood in particular appears as an independent risk factor of poor pain relief by analgesics. Given that PHQ9 scores were positively correlated with VAS and McGill scores, it is likely that depression-associated pain increase had caused insufficient analgesia. Alternatively, depression-related pathological alterations in neurotransmitters secretion or neural circuits might negatively affect the actions of gabapentinoids. In contrast to depression, there was no difference in anxiety between MSP and MRP. The probability to develop MRP did not increase with anxiety. This result indicated that depression and anxiety act differently on drug responsiveness, although the depression scores and anxiety scores had a statistically significant correlation (Spearman's correlation, rho = $0.161, p=0.0259)$. Depression is characterized by poor sleep quality [24]. In this study, we have observed a strong correlation between PHQ9 and PSQI scores (Spearman's correlation, rho = $0.502, p<0.0001)$. However, the poor sleep quality did not significantly affect the efficacy of analgesics, as advised by multivariate regression analysis.

Another interesting finding is the association of hypertension and drug responsiveness. After being adjusted by other characteristics, interestingly, hypertension was identified as a protective factor of MRP development. The association between hypertension and pain had been repeatedly reported in many single-center clinical studies. For instance, hypertension was correlated with lower prevalence of low back pain or osteoarthritis in Koreans [25], higher pain thresholds in participants with resolved functional abdominal pain [26], and lower chest pain scores in patients with acute myocardial 
infarction [27]. It is likely that hypertension was associated with lower pain scores among participants, and hence reducing the risk of MRP development. On the other hand, it was noted that most patients with hypertension had been prescribed medications targeting the renin-angiotensin-aldosterone system (RAAS modulators, for instance, angiotensin-converting enzyme inhibitors, angiotensin II receptor blockers and renin inhibitors). Recently, RAAS modulators have been attracting increasing attention for their potential application in the management of neurological disorders, including persistent neuropathic pain. A series of basic science research has demonstrated that both centrally and peripherally acting RAAS modulators, including ramipril and losartan, might attenuate pain hypersensitivities in different rodent models of neuropathic pain [28-30]. Therefore, it is likely that administration of antihypertensive medications contributed to the association of hypertension with decreased probabilities of medication resistance. Future animal and clinical studies on RAAS modulators-neuropathic pain relationship may provide novel regimens for the improvement of neuropathic pain management.

Clinical characteristics gathering at admission provided useful information for the identification of patients who may not benefit from pain medications. We fitted four machine learning models, namely, logistic regression, RF, SVM, and NB with these characteristics. Among the four models, SVM had the highest accuracy and ROC-AUC when we validated models with the test cohort. The sensitivity of current SVM classifier was as high as $93.5 \%$, suggesting that we may accurately identify almost all the patients with MRP using this model. Future studies, which recruit larger sample size (bigger training set), or new clinical characteristics, hold potential to further improve the performance of SVM models. Since all the clinical characteristics were collected following a standard procedure at admission, and no further clinical tests were required, the SVM classifier provided a highly cost-effective and efficient approach to screen MRP who demand regimens other than standard treatment. Apart from performance evaluation, we also assessed the "importance" of variables with the vip package. The variable that led to the greatest loss of model accuracy after being removed or permutated was considered the most important feature in the classifier. As demonstrated in the importance plot, the phase of disease, degree of skin lesions, diabetes, and hypertension were the top features that were required for accurate classification. For the backward stepwise logistic regression, removal of phase of disease, degree of skin lesions, and hypertension also caused a dramatic loss of AIC. Taken together, these three variables were considered the critical features for the diagnosis of MRP.

The present study also has some limitations. First, only patients who were over 50 years old were recruited from a single center in this cohort study. Therefore, the current machine learning models may not be competent in identifying younger patients who would develop MRP. Future studies with increased sample size and multi-center across a wider age range will improve the accuracies and applicability of prediction models. Second, our study had proposed that hypertension was a putative protective factor of MRP development. While administrations of antihypertensive agents were implicated in such effect of hypertension, we failed to collect the information of hypertension-related medications and test the implications of these medications. We would begin a new cohort study to explore the potential synergy between analgesics and antihypertensive agents.

\section{CONCLUSIONS}

The phase of diseases, degree of skin lesion, and depression are independent risk factors, whereas hypertension is a potential protective factor for developing analgesic resistance among patients with herpetic-related neuralgia. A SVM model adopting basic clinical features at admission could predict the outcome of analgesic responsiveness. 


\section{ACKNOWLEDGEMENTS}

Funding. This work and the journal's Rapid Service Fee were supported by grants from the National Natural Science Foundation of China (No. 82171378), and Shenzhen Municipal Science, Technology and Innovation Commission (No. JCYJ20180302144710880).

Authorship. All authors meet the International Committee of Medical Journal Editors (ICMJE) criteria for authorship for this article, take responsibility for the integrity of the work as a whole, and have given their approval for this version to be published.

Authors' Contributions. The authors' contributions were as follows: W Sun, X Liu and L Xiao were responsible for the concept and design of the study; J Zhou, W Sun, Y Liu, S Yang, S Wu, S Wang, D Xiong, I Ho, L Zhang, M Chan, $\mathrm{W} \mathrm{Wu}$, and $\mathrm{X}$ Liu were involved with experimental and analytical aspects of the manuscript; J Zhou, W Sun, X Liu and L Xiao performed data interpretation, presentation, and writing of the manuscript.

Disclosures. The authors, Jiehua Zhou, Wuping Sun, Yingzhi Liu, Shaomin Yang, Songbin Wu, Sashuang Wang, Donglin Xiong, Idy Hiuting HO, Lin Zhang, Matthew Tak Vai Chan, William Ka Kei Wu, Xiaodong Liu, Lizu Xiao have nothing to disclose.

Compliance with Ethics Guidelines. The present study was conducted in accordance with the Declaration of Helsinki and approved by the ethics committee of Shenzhen Nanshan People's Hospital and the 6th Affiliated Hospital of Shenzhen University Health Science Center (No. 2016041201). Written informed consent was obtained from all subjects.

Data Availability. The datasets generated during and/or analyzed during the current study are available from the corresponding author on reasonable request.
Open Access. This article is licensed under a Creative Commons Attribution-NonCommercial 4.0 International License, which permits any non-commercial use, sharing, adaptation, distribution and reproduction in any medium or format, as long as you give appropriate credit to the original author(s) and the source, provide a link to the Creative Commons licence, and indicate if changes were made. The images or other third party material in this article are included in the article's Creative Commons licence, unless indicated otherwise in a credit line to the material. If material is not included in the article's Creative Commons licence and your intended use is not permitted by statutory regulation or exceeds the permitted use, you will need to obtain permission directly from the copyright holder. To view a copy of this licence, visit http://creativecommons.org/licenses/by$\mathrm{nc} / 4.0 /$.

\section{REFERENCES}

1. Shah RA, et al. Shingrix for herpes zoster: a review. Skin Ther Lett. 2019;24(4):5-7.

2. Singh P, Silverberg NB, Silverberg JI. Outpatient healthcare utilization and prescribing patterns for herpes zoster in United States adults. Arch Dermatol Res. 2021;313(3):155-62.

3. Zerboni L, et al. Molecular mechanisms of varicella zoster virus pathogenesis. Nat Rev Microbiol. 2014;12(3):197-210.

4. Park SY, et al. Diagnostic usefulness of varicellazoster virus real-time polymerase chain reaction analysis of DNA in saliva and plasma specimens from patients with herpes zoster. J Infect Dis. 2017;217(1):51-7.

5. Yang F, et al. The epidemiology of herpes zoster and postherpetic neuralgia in China: results from a cross-sectional study. Pain Ther. 2019;8(2):249-59.

6. Wei S, et al. Analysis of the risk factors for postherpetic neuralgia. Dermatology. 2019;235(5): 426-33.

7. Hempstead MN, et al. Acute cortisol and behavior of dairy goat kids administered local anesthesia, topical anesthesia or systemic analgesia prior to cautery disbudding. Physiol Behav. 2020;222: 112942. 
8. Kopel J, Brower GL. Effectiveness of pregabalin as a secondary treatment for neuropathic pain from postherpetic neuralgia. Bayl Univ Med Cent Proc. 2020;33(3):469-70.

9. Mallick-Searle T, Snodgrass B, Brant JM. Postherpetic neuralgia: epidemiology, pathophysiology, and pain management pharmacology. J Multidiscip Healthc. 2016;9:447-54.

10. Huning S, et al. Therapy of herpes zoster and postherpetic neuralgia. Hautarzt. 2019;70(8):645-56.

11. Hunter P, Fryhofer SA, Szilagyi PG. Vaccination of adults in general medical practice. Mayo Clin Proc. 2020;95(1):169-83.

12. Zeng $P$, et al. Characterizing the structural pattern predicting medication response in herpes zoster patients using multivoxel pattern analysis. Front Neurosci. 2019;13:534.

13. Wei M, et al. EEG beta-band spectral entropy can predict the effect of drug treatment on pain in patients with herpes zoster. J Clin Neurophysiol. 2020. https://doi.org/10.1097/WNP.0000000000 000758.

14. Patel KV, et al. Evaluation of composite responder outcomes of pain intensity and physical function in neuropathic pain clinical trials: an ACTTION individual patient data analysis. Pain. 2018;159(11): 2245-54.

15. Aoyama K, et al. Determining associations and estimating effects with regression models in clinical anesthesia. Anesthesiology. 2020;133(3):500-9.

16. Kruppa J, et al. Probability estimation with machine learning methods for dichotomous and multicategory outcome: theory. Biom J. 2014;56(4):534-63.

17. Ehsani-Moghaddam B, et al. Mucopolysaccharidosis type II detection by Naive Bayes Classifier: an example of patient classification for a rare disease using electronic medical records from the Canadian Primary Care Sentinel Surveillance Network. PLoS One. 2018;13(12):e0209018.

18. Huang J, et al. Abnormal intrinsic brain activity and neuroimaging-based fMRI classification in patients with herpes zoster and postherpetic neuralgia. Front Neurol. 2020;11:532110.

19. Wiffen PJ, et al. Gabapentin for chronic neuropathic pain in adults. Cochrane Database Syst Rev. 2017;6:CD007938.
20. Derry S, et al. Pregabalin for neuropathic pain in adults. Cochrane Database Syst Rev. 2019;1: CD007076.

21. Marin M, et al. Risk factors for herpes zoster among adults. Open Forum Infect Dis. 2016;3(3):ofw119.

22. Stubbs B, et al. Depression and pain: primary data and meta-analysis among 237952 people across 47 low- and middle-income countries. Psychol Med. 2017;47(16):2906-17.

23. Rayner L, et al. Depression in patients with chronic pain attending a specialised pain treatment centre: prevalence and impact on health care costs. Pain. 2016;157(7):1472-9.

24. Segalas C, et al. Sleep disturbances in obsessivecompulsive disorder: influence of depression symptoms and trait anxiety. BMC Psychiatry. 2021;21(1):42.

25. Bae $\mathrm{YH}$, et al. Association between hypertension and the prevalence of low back pain and osteoarthritis in Koreans: a cross-sectional study. PLoS One. 2015;10(9):e0138790.

26. de la Coba $\mathrm{P}$, et al. Is resolution of chronic pain associated with changes in blood pressure-related hypoalgesia? Ann Behav Med. 2018;52(7):552-9.

27. Granot M, Dagul P, Aronson D. Resting blood pressure modulates chest pain intensity in patients with acute myocardial infarction. Pain Rep. 2019;4(3):e714.

28. Ogata $\mathrm{Y}$, et al. Involvement of spinal angiotensin II system in streptozotocin-induced diabetic neuropathic pain in mice. Mol Pharmacol. 2016;90(3): 205-13.

29. Hegazy N, Rezq S, Fahmy A. Mechanisms involved in superiority of angiotensin receptor blockade over ACE inhibition in attenuating neuropathic pain induced in rats. Neurotherapeutics. 2020;17(3): 1031-47.

30. Kaur P, Muthuraman A, Kaur J. Ameliorative potential of angiotensin-converting enzyme inhibitor (ramipril) on chronic constriction injury of sciatic nerve induced neuropathic pain in mice. J Renin Angiotensin Aldosterone Syst. 2015;16(1): 103-12. 